\title{
Absorción de minerales de una nueva fórmula láctea propuesta para el Programa Nacional de Alimentación Complementaria
}

\author{
Carlos Castillo D. ${ }^{1}$; Verónica Marín B. ${ }^{1}$; Gladys Barrera A. ${ }^{2}$; \\ Vivien Gattás Z. ${ }^{3}$; Ricardo Uauy D. ${ }^{1}$

\section{Mineral absortion in infants fed with a new modified cow's milk formula}

\begin{abstract}
Cow's milk has excessive calcium and phosphorus content and deficient amounts of zinc and copper, relative to the needs of normal infants. Calcium, phosphorus, magnesium, zinc, and copper absorption with a new cow milk formula which macronutrient and mineral compositions were adapted to comply with most normal intant's requirements. were studied in 9 male infants recovering from malnutrition in a closed nutritional recovery center. Patient's age was 3 to 10 months and their weightilength ratio was $\geqslant 85 \%$. Each infant was fed for six davs with unmodified powdered cow's milk diłuted to 10\%, "with sucrose 5\%. maltodextrin $1 \%$ and vegetable oil $1 \%$ added, and for another six days with modified cow's milk diluted to $15 \%$ plus maltodextrin $2,5 \%$. Metabolic balance was done in the last three days of each experimental perıod to every child. Modified milk had lower calcium, magnesium and phosphorus $(20 \%)$ and higher, copper $160 \%$ ) and zinc (25\%) contents (\%) than unmodified milk. Calcium absorption $(\%)$ was similar with both unmoditied arid modified milk $[49.0 \pm 14.2 \%$ vs $625 \pm 18.6 \%$ ); absolute calcium absorption with modified milk complied with accepted infant's needs. Apparent phosphorus absorption (54.3 $\neq 6.7$ with modified milk vs $86.8 \pm 9.1 \mathrm{mg} \cdot \mathrm{kg} \cdot \mathrm{d}$ with unmodified milk) should be enough for normal bone mineralization. Net magnesium absorption for modified milk was significantly lower than that of unmodifed milk $\left(3.5 \pm 1.2\right.$ vs $\left.0.1 \pm 2.2 \mathrm{mg} \cdot \mathrm{kg} \cdot \mathrm{d}_{;} \mathrm{p}<0.001\right)$. Copper absorption was deficient with unmodified miik but it was sufficient under modified milk $(95.4 \pm 13.3 \mu \mathrm{g} \cdot \mathrm{kg} \cdot$ d). Both formulae met recommended zınc allowances. The modified milk formula seems to berter meet mineral requirements of infants than unmodified powdered cow's milk.
\end{abstract}

\{Kev words: Infant tormula, cow's milk, mod.tied, mitieral absorption.\}

Los principales objetivos al desarrollar fórmulas lácteas, adecuadas a los requerimientos de minerales del primer año de vida, son promover un crecimiento y desarrollo psicomotor normal, asegurar una mineralización ósea adecuada y mantener una respuesta inmune 6 ptima $^{1,2}$. Entre los factores que dificultan en la actualidad una recomendación más exacta, en cuanto al aporte de minerales, están las múltiples interacciones entre minerales, o con otros nutrientes, que pueden modificar su biodisponibilidad o su

1. Instituto de Nutrición y Tecnología de los Alimentos, Universidad de Chile.

2. Enfermera Universitaria. Instituto de Nutrición y Tecnología de los Alimentos, Universidad de Chile.

3. Nutricionista. Instituto de Nutrición y Tecnología de los Alimentos, Universidad de Chile.

Financiado parcialmente por el Ministerio de Salud. metabolización y los insuficientes conocimientos acerca de los reales requerimientos de estos nutrientes. La leche de vaca, sin considerar la biodisponibilidad de los minerales, tiene un contenido teórico cercano al adecuado de zinc, excesivo de otros (calcio, fosforo), e insuficiente de otros (cobre, magnesio) ${ }^{1-10}$.

En la formulación de una leche de vaca en polvo modificada (LPM), que ha sido propuesta para incorporar en el Programa Nacional (chileno) de Alimentación Complementaria (PNAC), se han efectuado cambios en la concentración de algunos minerales y también de otros nutrientes, los cuales ya fueron analizados en publicaciones anteriores ${ }^{6,7,11,12}$. Ahora se describen $y$ analizan los resultados de estudios de ab. sorción de calcio, fósforo, magnesio, zinc y cobre en lactantes en recuperación nutricional, alimentados con leche de vaca modificada. 


\section{Material y Métodos}

Se seleccionaron lactantes con desnutrición primaria, hospitalizados para su tratamiento en un centro cerrado de recuperación nutricional, perteneciente a la Corporación para la Nutrición Infantil de Chile (CONIN), que tuvieran entre 3 y 10 meses de edad, peso de nacimiento superior a $2000 \mathrm{~g}$, relación peso/talla (P/T) sobre $85 \%$ del estándar OMS al momento de iniciar el estudio $y$ que estuviesen libres de enfermedades agudas o crónicas agregadas. Así se seleccionaton meve varones que, en el momento del balance, llevaban más de un mes de recuperación nutricional. Previo al injicio del estudio se solicitó la autorización de los padres mediante la firma de un consentimiento escrito e informado.

Todos los niños fueron sometidos a dos períodios sucesivos de estudio, asignados al azar, recibiendo en uno exclusivamente leche de vaca en polvo (LP) y, en el otro, leche de vaca en polvo modificada (LPM). La primera se reconstituyó al $10 \%$ con agregados de sacarosa $\$ \%$, maltosa-dextrina $1 \%$ y aceite $1 \%$, mientras que la LPM se reconstituyó al $15 \%$, con agregados dc maltosa-dextrina al $2.5 \%$, siendo ambas fórmulas isocalóricas (85 $\mathrm{kcal} / \mathrm{dl})$. En cuanto a la concentración de minerales de cada fórmula, a la LP no se le hizo cambios, de modo que su concentración fue la habi. tuat; en cambio en la LPM se disminuýn la concentración de calcio, magnesio y fósforo en aproximadamente $30 \%, y$ se aumentaron las concentraciones de cobre $(60 \%)$ y zinc $(25 \%$ ) (tabliz 1$)$. Se debe señalar que en lo que se refiere al zinc, la fórmula se diseño para tener una concentración de $5 \mathrm{mg} / \mathrm{l}$ y en la práctica resultó tener sólo $4 \mathrm{mg} / 1$, lo que no fue el ideal. Cada período de alimentación tuvo una duración de 6 días, correspondiendo los tres primeros a adaptacjón a la fórmula y los tres siguientes a balance. Durante el balance se efectuó recolección de orina de 24 horas por los tres días, mediante recolectores fijidos a la piel con aerosol adhesivo para evitar filtraciones; las deposiciones se recolectaron con papel filtro agregado a pañales desechables, de modo de evitar la inmovilización del niño. Al mismo tiempo se midieron cujdadosamente los volúmenes por diferencia en el peso de las mamaderas antes y después de cada alimentación. Las pérdidas de alimentación, así como de orina y heces, se registraton adecuadamente. Se tomaron atícuotas de alimentación y fecas, para medir el contenido de alcio, fósforo, magnesio, cobre y zinc mediante los siguientes métodos: para cobre, zinc, calcio y magnesio se utilizó espectrofotometría de absorción atómjca ${ }^{13}$, y para fóstoro, técrica fotocolorimétrica ${ }^{14}$. Como las muestras de deposiciones tomadas para el análisis del fósforo resultaron insuficientes en algunos casos, los resultados de absorción de este mineral corresponden sólo a seis de los nueve niños estudiados. También se tomaron muestras de sangre al final de cada balance, para la medición de los mismos minesales en el plasma, excepto para magnesio.

\section{Resultados}

Cobre. La figura 1 muestra que tanto la ingestión de cobre $(43,4 \pm 6,6$ para LP y $95,4 \pm$

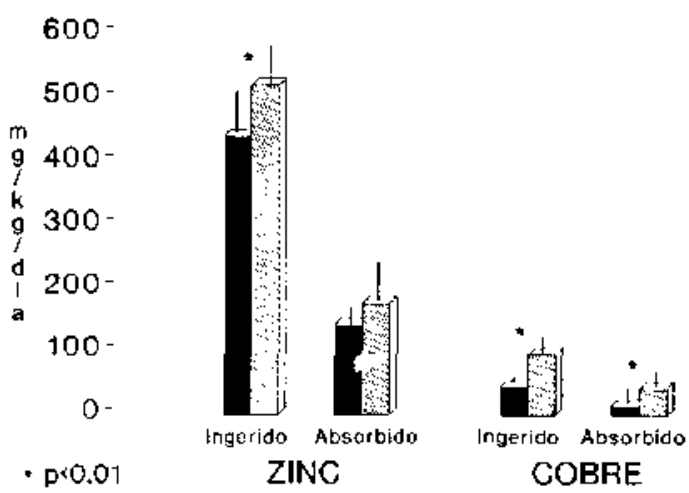

Figura 1: Ingesta y absorción de zinc y cobre, en dos períodos de balance efectuados con fórmulas en base a leche de vaca D, o leche modificada MIII, en lactantes $(n=9)$.

\section{Tabla 1}

Contenido de minerales de las fórmulas estudiadas (por dl de leche reconstituida) en relación a recomendaciones de ingesta diaria

\begin{tabular}{lccrc}
\hline $\begin{array}{c}\text { Contenido } \\
\text { ng/di }\end{array}$ & $\begin{array}{c}\text { Leche de vaca } \\
\text { en polvo }\end{array}$ & $\begin{array}{c}\text { Leche } \\
\text { modificada }\end{array}$ & \multicolumn{2}{c}{$\begin{array}{c}\text { Recomendación (RDA 1989) } \\
0 \cdot 5 \mathrm{~m}\end{array}$} \\
\hline Calcio & 95,0 & 75,0 & 400 & 600 \\
Fósforo & 68,0 & 48,0 & 300 & 500 \\
Magnesio & 9,2 & 6,0 & 40 & 50 \\
Zinc & 0,3 & 0,4 & 5 & 5 \\
Cobre & 29,0 & 68,0 & 500 & 650
\end{tabular}


$13,3 \mu \mathrm{g} \cdot \mathrm{kg} \cdot \mathrm{d}$ para la LPM), como su absorción $(13 \pm 16,5$ con $\mathrm{LP}$ y $41,8 \pm 13,6 \mu \mathrm{g} \cdot \mathrm{kg} \cdot \mathrm{d}$ con LPM) fueron significativamente mayores con LPM $(p<0,01)$. Cuando los lactantes recibieron $L P$, las absorciones aparentes resultaron negativas en dos casos $(-4 \mathrm{y}-19,2 \mu \mathrm{g} \cdot \mathrm{kg} \cdot \mathrm{d})$, hecho que no ocurrió con LPM (todos fueron positivos y con un minimo de $23,3 \mu \mathrm{g} \cdot \mathrm{kg} \cdot \mathrm{d}$ ). Al recibir LP hubo otros tres niños con absorciones menores de $10 \mu \mathrm{g} \cdot \mathrm{kg} \cdot \mathrm{d}$, lo que puede ser considerado insuficiente para un lactante en crecimiento. El porcentaje de absorción fue similar con ambas fórmulas $(36,8 \pm 30,2$ y $44,3 \pm$ $15,3 \%$ con LP y LPM, respectivamente). En cuanto al orden en que se efectuaron los balances, sólo en tres de los nueve casos se dio primero LPM; pero en los lactantes cuyos balances resultaron negativos para cobre, el orden en que recibieron cada fórmula no influyó, pues uno recibió primero LPM y el otro LP.

Zinc. A pesar que la ingesta de zinc con LPM fue significativamente mayor que con LP $(522,7$ $\pm 72,8 \mu \mathrm{g} \cdot \mathrm{kg} \cdot \mathrm{d}$ vs $442,4 \pm 67,3 \mu \mathrm{g} \cdot \mathrm{kg} \cdot \mathrm{d}$, $\mathrm{p}<0,01$ ) (figura 1) la absorción neta aparente fue similar con ambas fórmulas. Los balances con ambas fórmulas resultaron positivos, siendo el valor minimo absorbido con $L P$ de 38,3 $\mu \mathrm{g} \cdot \mathrm{kg} \cdot \mathrm{d}$ y $107,9 \mu \mathrm{g} \cdot \mathrm{kg} \cdot \mathrm{d}$ con LPM.

Calcio. El porcentaje absorbido de este mineral tendió a ser mayor con LPM (49 $\pm 14,2$ y $62,5 \pm 18,6 \%$ para LP y LPM, respectivamente, ns), lo que determinó que la absorción neta aparente fuera similar durante ambos períodos de estudio. La ingesta promedio diaria de calcio, mientras los niños recibian LP, excedió la recomendada para lactantes sanos (calcio ingerido $803 \mathrm{mg} \cdot \mathrm{d}$, recomendación RDA 1989 para lactantes de 6 a 12 meses: $600 \mathrm{mg} \cdot \mathrm{d}$ ), en cambio se mantuvo en el promedio con la LPM (figura 2).

Magnesio. La absorción aparente de magnesio fue mayor con la $\mathrm{LP}(7,49 \pm 2,1 \mathrm{mg} / \mathrm{kg} / \mathrm{d})$ que con la LPM $(3,5 \pm 1,2 \mathrm{mg} / \mathrm{kg} / \mathrm{d})$, lo que se relaciona con una ingesta significativamente menor con LPM (con LP 13,8 $\pm 1,9 \mathrm{mg} / \mathrm{kg} / \mathrm{d}$ y con LPM $8,5 \pm 1,2 \mathrm{mg} / \mathrm{kg} / \mathrm{d}$ ), p $<0,01$ ) (figura 2). Con la LPM todas las absorciones fueron positivas y adecuadas a la recomendación.

Fósforo. Los resultados analizados para este mineral están referidos a sólo seis balances; la ingesta fue mayor con $\mathrm{LP}$, pero los porcentajes de absorción se mantuvieron sin variación, lo que determino, como era de esperar, que la $a b$.

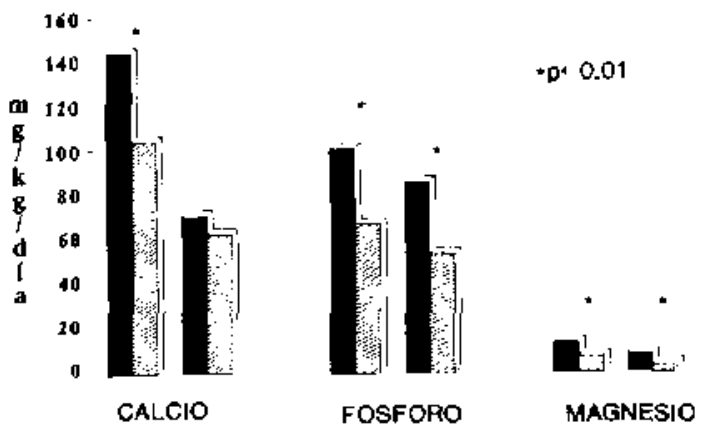

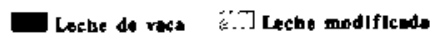

Figura 2: lngesta y absorción de calcio, tósforo y magnesio, en dos per íodos de balance efectuados con tórmulas en base a leche de vaca $\square$, 0 leche modificada MII, en lactantes ( $\mathrm{n}=9$ para Ca y $\mathrm{Mg}, \mathfrak{n}=6$ para $\mathrm{P}$ ).

sorción neta aparente fuera significativamente menor con LPM.

Las concentraciones plasmáticas de los mi. nerales analizados se ubicaron dentro de los rangos normales para la edad, y sin diferencias significativas durante ambos períodos (tabla 2).

\section{Comentario}

El propósito fundamental de este estudio fue evaluar la absorción intestinal de minerales con una fórmula lảctea que, teóricamente, aporta estos nutrientes en cantidad y proporciones más cercanas a las recomendadas para lactantes sanos menores de un año. Los porcentajes de absorción de todos los minerales no mostraron diferencias significativas, en contraste con lo reportado en la literatura, con el uso de fórmulas con distintas concentraciones de ellos, lo cual puede deberse, en primer lugar, a la posibilidad que los tres días de estabilización empleados en el estudio sean insuficientes para observar cambios, especialmente en lo que respecta a absorción activa del mineral, observándose una tendencia a aumentar el porcentaje absorbido, al disminuir la ingesta, sólo en caso del calcio. En segundo término, ta concentración de calcio y fósforo se modificó, en la nueva fórmula, sin cambiar significativamente la relación Ca: $\mathrm{PO}_{4}$, que parece tener mayor importancia que las concentraciones absolutas de cada mineral 
Tabla 2

Concentraciones plasmáticas de cobre, zinc, calcio, fósforo y magnesio en lactantes alimentados con fótmula en base a leche de vaca o leche de vaca modifícada

\begin{tabular}{|c|c|c|c|}
\hline & & L.P. & L.P.M. \\
\hline Cupremia & $(\mu \mathrm{g} / \mathrm{d})$ & $124,0 \pm 43,4$ & $141,0 \pm 35,1$ \\
\hline Zincemia & $(\mu \mathrm{g} / \mathrm{dl})$ & $92,1 \pm 18,0$ & $94,3 \pm 16,5$ \\
\hline Calcemia & (mg/dl) & $8,8 \pm 1,0$ & $8,9 \pm \quad 0,7$ \\
\hline Fosfemia & $(\mathrm{mg} / \mathrm{dl})$ & $5,9 \pm 0,7$ & $6.1 \pm 0.6$ \\
\hline
\end{tabular}

L.P.: Leche de vaca en polvo no modificada.

L.P.M.: Leche de vaca en polvo modificada.

en determinar cambios en la fracción absorbi$\mathrm{da}^{2-6}, 15-21$. Además, en el caso del fósforo, su retención se regula principalmente en los riñones, de modo que tienen que ocurrir cambios muy importantes en la ingestión para que se modifique su porcentaje de absorción. También debe considerarse, en la interpretación de los resultados, que este estudio se realizó en lactantes desnutridos en recuperación, lo cual puede inducir aumentos en la absorción de los minerales, especialmente de calcio, zinc y co$\mathrm{bre}^{2,4}$. Asimismo, el estado nutricional previo para estos minerales sólo lo conocemos parcialmente a través de sus concentraciones plasmáticas, en estos niños, y, en el caso del calcio, sus valores promedios correspondian a lo normal bajo, to que hace plantear un déficit anterior $y$, por lo tanto, una absorción activa máxima independiente del aporte. Este mismo planteamiento es válido para el cobre, cuyas concentraciones plasmáticas también resultaron levemente bajo lo normal, pues los lactantes en recuperación nutricional tienen mayor tiesgo de deficiencia de cobre ${ }^{1,2,22}$. Que las concentra. ciones de zinc estèn dentro de lo normal, no asegura adecuada nutrición de este mineral, pues sabemos que ellas no constituyen un criterio suficiente de evaluación ${ }^{2}$.

Las ingestas absolutamente insuficientes y absorciones negativas de cobre con LP confirman la necesidad de modificar las fórmulas lácteas actualmente en uso para ofrecer concentraciones de $0,6 \mathrm{mg} / 1$ como las recomendadas por la Academia Americana de Pediatría y usada en este estudio, previniendo los riesgos que hemos observado en estudios anteriores, como son una menor velocidad de crecimiento, leucopenia, neutropenia y alteraciones en la función inmune 22,23 .

La ingesta promedio de zinc alcanzada con las dos fórmulas fue insuficiente para ambas $(2,5 \mathrm{mg} \cdot d$ con LP y $3 \mathrm{mg} \cdot d$ con LPM), con respecto a la recomendada de $5 \mathrm{mg}$. d para lac. tantes sanos. Ingestas como la descrita influyen en menor recuperación nutricional y comprometen la función inmunitaria ${ }^{24}$, por esto es necesa. rio insistir en el uso de fórmulas en concentraciones de $5 \mathrm{mg} / \mathrm{l}$ como las del diseno original de la fórmula. A pesar de esto, la absorción mínima alcanzada con LPM es adecuada para nif̃os eutróficos, y el porcentaje de absorción encontrado se ubica dentro del margen normal alto.

Con las modificaciones de la nueva fórmula en análisis se pretendía reducir la ingestión de calcio, disminuyendo su absorcion a tasas que permitiesen una mineralización ósea adecuada, $\sin$ los riesgos de los aportes excesivos, simila. res a los observados en hiperparatiroidistno o la hipercalciuria idiopática ${ }^{4}$. Los porcentajes de absorción de calcio encontrados en estos lactantes durante la alimentación con ambas fórmulas fueron superiores a los habituales con leche de vaca, lo que, como ya se señaló, puede de. berse al tipo de pacientes en que se realizaron las mediciones. La fórmula modificada mostró reducciones de la absorción de fósforo que permiten la mineralización ósea adecuada sin riesgo de exceso.

Dos niños mostraron absorciones de magnesio inferiores a 40\% de los aportes cuando recibian LPM, lo que podría deberse al corto periodo de adaptación empleado. 
Estos resultados sugieren que la fórmula láctea modifícada permite ingestión y absorción de los minerales evaluados, concordantes con las recomendaciones aceptadas habitualmente para lactantes sanos $\mathrm{s}^{3,4,6}$.

\section{Resumen}

La leche de vaca tiene un contenido excesivo de calcio $y$ fósforo $e$ insuficiencia de zinc $y$ cobre. En la formulación de una leche modificada se han cambiado las concentraciones de estos minerales, para acercarlas a las recomen. daciones normales. Se estudió la absorción de estos nutrientes en 9 lactantes hospitalizados en recuperación nutricional ( $\mathrm{P} / \mathrm{T}$ : $\geqslant 85 \%$ ). Cada niño recibió leche de vaca y leche modificada por 6 dias, usándose los tres últimos días de cada periodo para balance. Encontramos ingestas inadecuadas de cobre $(50 \%)$ y zinc (50\%) y absorciones bajas de cobre $(13 \mu \mathrm{g} \cdot \mathrm{kg} \cdot \mathrm{d}$ ) en relación a las correspondientes recomendaciones, cuando se empleó Jeche de vaca no modi. ficada. Con la leche modificada sólo la ingesta de zinc $(60 \%)$ fue insuficiente. Las ingestas de calcio (140\%) y fósforo (117\%) fueron excesi. vas mientras los niños recibían leche de vaca, pero adecuadas con leche modificada. La leche modificada empleada en este estudio asegura ingestas y absorciones más adecuadas que la leche de vaca no modificada para los minerales eva. luados.

(Palabras claves: Fórmula láctea artificial, leche de vaca modificada, ingesta, absorción, minerales, calcio, cobre, fósforo, zinc, magnesio.)

\section{Agradecimientos}

Agradecemos sinceramente la colaboración prestada por el personal de CONIN (ex Centro Manuel Montt) $y$ al señor Patricio Segoyia, por los análisis de laboratorio

\section{Referencias}

1. Lönnerdal B, Bell JG. Keen CL: Copper absorption from human milk, cow's milk, and infant formulas using a suckling rat model. Am J Clin Nutr 1985; 42: $836-844$

2. Milner $I A$ : Trace minerals in the nutrition of children. J Pediate 1990: 117: s147-155
3. De Vizia B, Fomon SJ, Nelson $S E$, Edwards $B E$, Ziegler $E E$ : Effect of dietary calcium on metabolic balance of normal infants. Pediatr Res 1985; 19: $800-806$

4. Recommended Dietary Allowances 10th Edition. National Research Council. National Academy Press Wishington, D.C. 1989.

5. Forbes RM, Erdman JW, Porker HM, Kondo $H$, Refelsen SH. Bioavailability of zinc in coagulated soy protein (tofu) to rats and effect of dietary calcium at a constant phytate: zinc ratio. J. Nutr 1983; 113: 205-210

6. King $J$, De Pablo $S$, Montes de Oca $F$, Uauy $R$ : Formulación. fabricación y evaluación de una Fórmula láctea para el Prograna de Alimentación Complementaria en Chile. Rev Chil Pediatr 1991; 62: 17.7 .

7. Castillo $C$, Barrera $G L$, Gazás V, Riumalló $J$, Jorpo $S$, Alliende $F$ : Nueva fórmula láctea para el Programa Nacional de Alimentación Complementaria: balance nitrogenado y calórico. Rev Chil Pediatr $1991: 62 \div 8+13$

8. OMS: Medición del cambio del estado nutricional, Publicaciones OMS. Gincbra 1983.

9. Hillman L: Mjnesal and vitamin D adequacy in infants fed human milk or formula between 6 and 12 months of age. I Pediatr 1990:117: s1 34-s142

10. ESPGAN: Committee on Nutrition, Guidelines on [ntant Nutrition. III. Recommendations for infant feeding. Acta Ped Scand 1982: Suppl 302.

11. Jury GL, Castilks $C D$, Atalah E, Puentes $k$, Riumollo $\mathrm{J}$ : Crecimiento, aceptación y tolerancia con una nueva fórmula táctea. Rev Chil Pediatr $1991: 62: 8793$.

12. Hertrampf $E$. Pizarto $F$, Peteyra $A$, Vega $U$ : Nueva fórmula láctea. Biodisponibilidad del hieIro $y$ efecto en la prevención de su deficiencia en lactantes. Rev Chil Pediatr 1991;62: 94-98.

13. Fernández EJ. Kahn HL: Clinical method for atomic absorption espectroscopy Clin Chem Newslett $1971 ; 3: 24-28$.

14. Boliz DF. Mellon MG: Spectuphotometric determination of phosphorus as molybdiphosphoric acid. Anal chem 1948:20:749.

15. Greger Jl, Snedeker SM: Effect of dietary protein and phosphorus levels on the utilization of zinc. copper, and manganese by adult males. I Nutr 1980; 110: 2243-53.

16. Clark 1: Metabolic interrelations of calcium, mag nesium, and phosphate. Am J Physiol 1969: 217:871-877.

17. Kate L, Hamilton JR: Fat absorption in infants of birth weight less than $1,300 \mathrm{~g}$. I Pediatr 1974; $85: 608-614$.

18. Clark I: Importance of dietary $\mathrm{Ca}: \mathrm{PO}_{4}$ ratios on skeletal $\mathrm{Ca}$, Mg, and $\mathrm{PO}_{+}$metabolism Am J physiol 1969;217: 865-870.

19. Noveh $Y$, Hazani A, Berant M: Copper deficiency with cow's milk diet. Pediatrics 1981 ; 68: 397 . 400

20. Goto $S h$, Stugai $T$ : Effect of excess calcium intake on absorption of nitrogen, fat, phosphorus and 
calcium in adult rats. The use of organic catcium salt Nutr Rep lnt 1975;11:49-54.

21. Norman OA. Fordiran IS, Brinkley IJ, Zerwekh $J E$, Nicar MJ, Strowig SM, Pak ChrC: Jejunal and ileal adaptation to alterations in dietary calcium. J Clin lnvest 1981;67:1599-1603.

22. Castillo-Durán C, Uauy R: Copper deficiency impairs growth of infants recovering from malnutrition. Am J Clin Nutr 1988: 47: 710-714.

23. Heresi $G$, Castillo-Durán C, Muñoz C, Arévalo $M$, Schlesinger $I:$ : Phagocy tosis and immunoglobulins levels in hypocupremic infants. Nutr Res 1985; $5: 1327-34$.
24. Castllo-Durán $C$, Heresi $G$, Fisberg $M$, Vouy $R$ : Controlled trial of zinc supplementation during recovery from malnutrition. Effects on growth and immune function. Am J Clin Nutr 1987; 45: $602-608$.

25. Giles $M M$, Laing IA, Elton RA, Robins JB, Sanderson M, Hume R. Magnesium metabolism in preterm infants: Effects of calcium, magnesium, and phosphorus and of post-natal and gestational age. J Pediatr 1990; 117: 147-154.

26. Shils $M E$ : Magnesium in healt and disease. Ann Rev Nut $1988: 8: 429-460$. 